\title{
PHYSICAL AND FUNCTIONAL COMPLICATIONS AFTER SURGICAL TREATMENT FOR BREAST CANCER
}

Amanda Estevão1, Cinira Assad Simão Haddad', Samantha Karlla Lopes de Almeida Rizzi', Afonso Celso Pinto Nazário', Gil Facina ${ }^{1}$

${ }^{1}$ Universidade Federal de São Paulo - São Paulo (SP), Brazil.

Introduction: The ideal treatment for breast cancer should be carried out by a multidisciplinary team, aiming at the global approach of the patient. It is usually a prolonged process and can cause relevant changes in the lives of patients, such as limited shoulder movement, pain, lymphedema, adherence scarring, sensitive changes, phlebitis, among others. Physical therapy intervention plays an essential role and can prevent and minimize complications, besides enabling functional recovery. It is extremely important that the physical therapy service is aware of the prevalence of the main physical and functional complications and the impact of its intervention. Objective: To assess the main physical and functional complications in the post-surgical treatment of breast cancer. Methods: This is a cross-sectional study in which we analyzed the medical records of patients treated in the Physical Therapy Ambulatory from 2011 to 2016. We obtained data about the surgery, prevalence of pain, changes in perimeter, movement amplitude, evaluation of scapulohumeral rhythm and presence of axillary cording. Results: We assessed the data of 703 patients, whose mean age was 56 years; $50.1 \%$ were married, and body mass index pointed to overweight (mean: $26.8 \mathrm{~kg} / \mathrm{m}^{2}$ ). Sedentary lifestyle was prevalent $(73.7 \%)$. The main surgery performed was quadrantectomy, followed by mastectomy. Pain was present, however, in extremely low levels (mean of pain in the Visual Analog Scale on the seventh postoperative day was 2.55); movement amplitude restrictions were higher in the beginning, and functionally reversed during follow-up; scapular dysfunction is also little prevalent (9.9\%), with high percentage of long term resolution. The incidence of axillary cording was high (87.9\%), which requires special attention. Conclusion: The study allowed to observe the main physical and functional complications after breast cancer treatment, and show the importance and necessity of early physical therapy. 